\title{
Prostatic Artery Embolization: Influence of Cone-Beam Computed Tomography on Radiation Exposure, Procedure Time, and Contrast Media Use
}

\author{
F. Bürckenmeyer ${ }^{1}$ I. Diamantis ${ }^{1}$ - T. Kriechenbauer ${ }^{1}$ T. Lehmann ${ }^{2}$ • \\ T. Franiel ${ }^{1}$ - A. Malouhi ${ }^{1} \cdot$ M. O. Grimm ${ }^{3}$ - U. Teichgräber ${ }^{1} \cdot$ R. Aschenbach ${ }^{1}$ (D)
}

Received: 16 October 2020/Accepted: 23 January 2021/Published online: 3 March 2021

(C) The Author(s) 2021

\begin{abstract}
Purpose To evaluate the effect of cone-beam computed tomography (CBCT) on radiation exposure, procedure time, and contrast media $(\mathrm{CM})$ use in prostatic artery embolization (PAE).

Materials and Methods Seventy-eight patients were enrolled in this retrospective, single-center study. All patients received PAE without (group A; $n=39$ ) or with (group $\mathrm{B} ; n=39$ ) CBCT. Total dose-area product $\left(\mathrm{DAP}_{\text {total }} ; \mathrm{Gycm}^{2}\right)$, total entrance skin dose $\left(\mathrm{ESD}_{\text {total }}\right.$; $\mathrm{mGy})$, and total effective dose $\left(\mathrm{ED}_{\text {total }} ; \mathrm{mSv}\right)$ were primary outcomes. Number of digital subtraction angiography (DSA) series, CM use, fluoroscopy time, and procedure time were secondary outcomes. PAE in group A was performed by a single radiologist with 15 years experience, PAE in group B was conducted by four radiologists with 4 to 6 years experience.

Results For groups A vs. B, respectively, median (IQR): $\mathrm{DAP}_{\text {total }} 236.94$ (186.7) vs. 281.20 (214.47) $\mathrm{Gycm}^{2}(-$ $p=0.345) ; \mathrm{ED}_{\text {total }} 25.82$ (20.35) vs. 39.84 (23.75) $\mathrm{mSv}$ $(p=<0.001) ; \mathrm{ESD}_{\text {total }} 2833$ (2278) vs. 2563 (3040) $\mathrm{mGy}(p=0.818)$; number of DSA series 25 (15) vs. 23 $(10)(p=0.164)$; CM use 65 (30) vs. 114 (40) $\mathrm{mL}(p=<$ 0.001 ); fluoroscopy time 23 (20) vs. 28 (25) $\min (p=$ $0.265)$, and procedure time $70(40) \mathrm{vs} .120(40) \min (p=$
\end{abstract}

R. Aschenbach

rene.aschenbach@med.uni-jena.de

1 Department for Diagnostic and Interventional Radiology, University Hospital Jena, Am Klinikum 1, 07747 Jena, Germany

2 Center for Clinical Studies, University Hospital Jena, Jena, Germany

3 Clinic for Urology, University Hospital Jena, Jena, Germany
$<0.001)$. Bilateral PAE was achieved in 33/39 (84.6\%) group A and 32/39 (82.05\%) group $\mathrm{B}(p=0.761)$, all other patients received unilateral PAE. There were no significant differences between clinical parameters and origins of the prostatic arteries (PA) $(p=0.206-1.00)$.

Conclusion Operators with extensive expertise on PAE may not benefit from addition of CBCT to DSA runs, whereas for operators with less expertise, CBCT when used alongside with DSA runs increased the overall radiation exposure.

Keywords Prostatic artery embolization - Radiation exposure - Cone-beam computed tomography $\cdot$ Dosearea product $\cdot$ Contrast media

\section{Introduction}

Prostatic artery embolization (PAE) is a minimally invasive procedure that is a new option for treating patients with lower urinary tract symptoms caused by benign prostatic hyperplasia [1]. PAE has advantages over traditional surgical therapies because it is minimally invasive with high rates of technical success and results in improved urinary flow rates and quality of life [2-8]. However, because of its complexity, the procedure demands a high level of investigator skill and long fluoroscopy times as well as angulated views, which can lead to higher radiation exposure for both staff and patients [1]. The longer fluoroscopy times and more angulated views are often related to determine the origins of the prostatic arteries (PA) and to effectively cannulate or evaluate the distal PA anatomy. 
One approach to this is the use of intraprocedural conebeam computed tomography (CBCT). CBCT could replace digital subtraction angiography (DSA) to identify the PA anatomy and to help treatment planning during PAE. On the other hand, CBCT increases the radiation exposure of the patient and requires more contrast media $(\mathrm{CM})$ to visualize the vascular anatomy [9-12]. Although PAE procedures are increasingly performed, limited data are available yet to determine the additional radiation dose related to CBCT in patients. The aim of this study was to compare two approaches to PAE-with and without intraprocedural CBCT.

\section{Methods}

The study protocol was conducted in accordance with the Declaration of Helsinki and approved by the local institutional review board. All patients provided written informed consent before the procedure. All patients referred to PAE between January 2015 and May 2018 were retrospectively included in this single-center observational study. Our study group previously published overall data from this cohort [13]. To give a more detailed analysis how CBCT influence the radiation exposure and CM use, this study is a sub-group analysis comparing patients allocated into two groups, group A (procedure without $\mathrm{CBCT}$ ) and group $\mathrm{B}$ (procedure with CBCT). The decision, if CBCT performed was at the discretion of the interventionalist. All interventions were performed using Artis ZeeGo Q system (Siemens, Erlangen, Germany). Except CBCT, all interventionalists used neutral planes and oblique planes according the anatomical needs. CM injection for DSA was performed by hand; $10 \mathrm{~mL} /$ injection via guiding catheter and $1 \mathrm{~mL} /$ injection via microcatheter. CBCT was performed using a 7 -s rotational scan of $180^{\circ}$ with an image acquisition of 60 frames per second. Dataset was transferred to a dedicated workstation (Leonardo, Siemens, Erlangen, Germany) to calculate planar datasets and 3D renderings. As described earlier, CBCT was performed placing the catheter in the distal aorta to evaluate both internal iliac arteries and the origins of the PA. $90 \mathrm{~mL}$ of $\mathrm{CM} /$ saline mixture $(60 \% / 40 \%$ : total amount of CM $54 \mathrm{~mL})$ was injected with a delay of $4 \mathrm{~s}$ and a flow of $8 \mathrm{~mL} / \mathrm{sec}$ [5]. No computed tomography angiography (CTA) or magnetic resonance angiography (MRA) was performed prior to embolization. PAE in group A was performed by a radiologist with 15 years of experience in embolization therapy. Patients included in group B were investigated by four radiologists, each with 4 to 6 years of experience in interventional therapies. To reduce the bias in experience and with response to the learning curves according the procedure, we excluded the first 50 patients for all interventionalists based on the results of our former study [13].

\section{Study Outcome Measurements}

For all PAE, entrance skin dose of fluoroscopy $\left(\mathrm{ESD}_{\text {fluoro }}\right)$, total entrance skin dose $\left(\mathrm{ESD}_{\text {total }}\right)$, entrance skin dose of DSA runs, $\left(\mathrm{ESD}_{\text {series }}\right)$ dose-area product of fluoroscopy $\left(\mathrm{DAP}_{\text {fluoro }}\right)$, total dose-area product $\left(\mathrm{DAP}_{\text {total }}\right)$, dose-area product of DSA runs $\left(\mathrm{DAP}_{\text {series }}\right)$, number of DSA runs series, and, if performed, CBCT-specific DAP and ESD $\left(\mathrm{DAP}_{\mathrm{CBCT}} ; \mathrm{ESD}_{\mathrm{CBCT}}\right)$ were gathered using examination reports from the picture archiving and communication system. Procedure time, $\mathrm{CM}$ use, and bilaterality of embolization were obtained from the radiological information system.

$\mathrm{DAP}_{\text {total }}\left(\mathrm{Gy} / \mathrm{cm}^{2}\right), \mathrm{ESD}_{\text {total }}(\mathrm{mGy})$, and effective dose (ED, $[\mathrm{mSv}]$ ) were the primary measurement outcomes. To calculate the ED from DAP of DSA series (2D imaging) in PAE, we used a conversion coefficient of $0.109 \mathrm{mSv} /$ $\mathrm{Gycm}^{2}$ based on Struelens et al. [14]. To estimate ED from $\mathrm{DAP}_{\mathrm{CBCT}}$, a conversion coefficient of $0.13 \mathrm{mSv} / \mathrm{Gycm}^{2}$ was used [15-17].

Secondary outcomes were the number of DSA series, CM use, fluoroscopy time, and procedure time, as well as $\mathrm{ESD}_{\text {fluoro }}, \mathrm{ESD}_{\text {series }}$; $\mathrm{DAP}_{\text {series }}$ and $\mathrm{DAP}_{\text {fluoro }}$. Procedure time was defined as the "table time" of the patient from start to end of the procedure. To compare both groups, age, body mass index, prostate volume, international prostate symptom score (IPSS), international index of erectile function, international index of erectile function erectile domain, quality of life, and peak urinary flow rate were obtained from patients' clinical records.

To reflect the complexity of the procedures, angiograms were scored side-by-side according to de Assis et al. [18]. All interventionalists scored the angiograms in consensus.

\section{Statistical Analysis}

Continuous variables are reported as median and interquartile range (IQR) or as means with standard deviations ( \pm SD). Primary outcome parameters were compared using Mann-Whitney $U$ test for unpaired independent datasets. Categorial values were compared by two-sided Fisher's exact test. To compare the rate of bilaterality, we used cross-table chi-square test. The threshold $p$ value for significance was $p=0.05$. Analyses were performed using SPSS software (version 26, IBM Corp., Armonk, NY, USA). 


\section{Results}

Thirty-nine patients were included in each group, and patients' baseline characteristics are summarized in Table 1. We found no significant difference in clinical parameters between the two groups ( $p=0.206-0.645$ ). The angiographic characteristics according to the PA origins are summarized in Table 2.

For primary outcome measurements, median (IQR) DAP $_{\text {total }}$ was 236.94 (186.7) $\mathrm{Gycm}^{2}$ and 281.20 (IQR 214.47) $\mathrm{Gycm}^{2},(p=0.345)$, resulting in median $\mathrm{ED}_{\text {total }}$ of 25.82 (IQR 20.35) $\mathrm{mSv}$ and 39.84 (23.75) $\mathrm{mSv}$ for group A and group $\mathrm{B},(p=<0.001)$, respectively. Median (IQR) $\mathrm{ESD}_{\text {total }}$ was 2833 (2278) mGy for group A versus 2563 (3040) $\mathrm{mGy}$ for group $\mathrm{B}(p=0.818)$. Median (IQR) $\mathrm{DAP}_{\mathrm{CBCT}}$ and $\mathrm{ESD}_{\mathrm{CBCT}}$ were 70.76 (13.22) and 232 (43.75) in group $\mathrm{B}$, resulting in an $\mathrm{ED}_{\mathrm{CBCT}}$ of 9.19 (IQR 1.71) $\mathrm{mSv}$.

For secondary outcome measurements, the median (IQR) number of DSA series was 25 (15) in group A and 23 $(10)$ in group B $(p=0.16)$. The median (IQR) amount of $\mathrm{CM}$ for the whole procedure was $65(30) \mathrm{mL}$ in group A and $114(40) \mathrm{mL}$ in group $\mathrm{B}(p \leq 0.001)$. The median (IQR) fluoroscopy time for group A and B was 23 (20) min and $28(20) \min (p=0.265)$, respectively, and the median (IQR) procedure time was 70 (40) vs. 120 (40) min, which was significantly different $(p \leq 0.001)$. ESD $_{\text {flouro }}$ was 252 (282) mGy for GroupA and 634 (603) mGy for Group B $(p=<0.001), \mathrm{DAP}_{\text {fluoro }}$ was 18.88 (24.82) $\mathrm{Gycm}^{2}$ for Group A and 28.98 (30.44) $\mathrm{Gycm}^{2}$ for Group B $(p=0.009) . \mathrm{ESD}_{\text {series }}$ and $\mathrm{DAP}_{\text {series }}$ were 2585 (2047) mGy vs. 2245 (2675) mGy for GroupA vs.B $(p=0.5)$ and 226.75 (174.5) $\mathrm{Gycm}^{2}$ vs. 243.46 (206.31) $\mathrm{Gycm}^{2}$ ( $p=0.593$ ). Bilateral embolization was achieved in $33 / 39$ $(84.6 \%)$ patients in group A and in $32 / 39(82.05 \%)$ patients in group $\mathrm{B}(p=0.761)$. All other patients received a unilateral PAE, there were no patients with PAE technical failure. Results are summarized in Table 3.

\section{Discussion}

Various reports recommend CBCT in PAE to define origins of the PA, to save CM, limit radiation exposure to patient and staff, and to avoid nontarget embolization and to achieve bilateral embolization [5, 9-11, 19-21]. CBCT was considered helpful in determination of PA origin and in identification of the artery itself, because this remains one of the most challenging parts of the procedure $[5,21]$. Although CBCT is considered helpful in identifying PA origin, the additional radiation dose resulting from extra three-dimensional imaging acquisition during PAE is important [17]. Enderlein et al. reported that the combination of CBCT and DSA results in a reduced need for contrast agent and a shortening of fluoroscopy times [22]. In our study, mean fluoroscopy times were $27.46 \mathrm{~min}$ $( \pm 14.43)$ for group $A$ and $29,74 \mathrm{~min}( \pm 13,44)$ for group $\mathrm{B}(p=0.265)$. Although CBCT was available for group B, the fluoroscopy time has no significant difference. This may reflect the lesser experience of the interventionalists in group B. The more frequent use of magnified and angulated views led to a significantly higher $\operatorname{ESD}(p=<0.001)$ and DAP $(p=0.009)$ for fluoroscopy in Group $\mathrm{B}$, despite similar fluoroscopy times between groups. Further, additional use of CM in group B was mostly due to the CBCT performed in the aorta before PAE. The number of DSA series was almost equal and did not differ significantly between groups. Distribution of PA origins was comparable in both groups with around $65 \%$ for Type I which is the most challenging type for cannulation [18]. Group A achieved $84.6 \%$ of bilateral PAE compared with $82.05 \%$ in group $\mathrm{B}, p=0.761$. Although this difference was not significant, it is a clear indicator that PAE is possible with a
Table 1 Patients characteristics $(\mathrm{n}=78)$

\begin{tabular}{|c|c|c|c|c|c|}
\hline & \multicolumn{2}{|c|}{ Group A $(n=39)$} & \multicolumn{2}{|c|}{ Group B $(n=39)$} & \multirow[t]{2}{*}{$p$-value } \\
\hline & Median [IQR] & Mean $[ \pm \mathrm{SD}]$ & Median [IQR] & Mean $[ \pm \mathrm{SD}]$ & \\
\hline Age, years & $66.8[11,35]$ & $67.2[ \pm 7.8]$ & $67.6[10,26]$ & $69.1[ \pm 6]$ & 0.303 \\
\hline IPSS & $25[8]$ & $24[ \pm 6]$ & $23[9]$ & $23[ \pm 7]$ & 0.334 \\
\hline IIEF 5 & $17[11]$ & $15.6[ \pm 7.6]$ & $16[16]$ & $14.8[ \pm 7,7]$ & 0.645 \\
\hline IIEF-EF & $21[12]$ & $19.3[ \pm 9.1]$ & 19 [16] & $18.2[ \pm 9,1]$ & 0.552 \\
\hline QoL & $5[2]$ & $4.8[ \pm 1.25]$ & $5[1]$ & $4.56[ \pm 1,25]$ & 0.283 \\
\hline Peak urinary flow rate & $10[5]$ & $10.1[ \pm 3.8]$ & $9[6]$ & $9.6[ \pm 4]$ & 0.545 \\
\hline Prostate volume, $\mathrm{ml}$ & $60[36]$ & $66.7[ \pm 7.8]$ & $62[37]$ & $72.2[ \pm 39,6]$ & 0.401 \\
\hline Body mass index, $\mathrm{kg} / \mathrm{m}^{2}$ & $26.5[1,7]$ & $26.9[ \pm 1.15]$ & $27.2[1,5]$ & $27.2[ \pm 0.9]$ & 0.206 \\
\hline
\end{tabular}

IPSS international prostate symptom score, IIEF-5 international index of erectile function, IIEF-EF international index of erectile function erectile domain, QoL quality of life 
Table 2 Angiographic characteristics $(\mathrm{n}=78)$

\begin{tabular}{llll}
\hline & Group A & Group B & $p$ value \\
\hline Number PA origin Type I & $50 / 78(64.1 \%)$ & $51 / 78(65.38 \%)$ & 1.000 \\
Number PA origin Type II & $13 / 78(16.66 \%)$ & $12 / 78(15.38 \%)$ & 1.000 \\
Number PA origin Type III & $3 / 78(3.85 \%)$ & $5 / 78(6.41 \%)$ & 0.719 \\
Number PA origin Type IV & $12 / 78(15.38 \%)$ & $10 / 78(12.82 \%)$ & 0.819 \\
Number PA origin Type V & $0 / 78(0 \%)$ & $0 / 78(0 \%)$ & 1.000 \\
\hline
\end{tabular}

PA prostate artery, origins definded according to Assis et al

Type I: origin from the anterior division of internal iliac artery in a common trunk with the superior vesical artery (SVA)

Type II: origin from anterior division of internal iliac artery, inferior to SVA

Type III: origin from obturator artery

Type IV: origin from internal pudendal artery

Type V: less common origins

Table 3 Results

\begin{tabular}{|c|c|c|c|c|c|c|c|}
\hline & \multicolumn{3}{|c|}{ Group A $(n=39)$} & \multicolumn{3}{|l|}{ Group B $(n=39)$} & \multirow[t]{2}{*}{$p$ value } \\
\hline & Median[IQR] & $\begin{array}{l}\text { Mean }[\mathrm{SD}] 95 \% \\
\text { CI }\end{array}$ & $\begin{array}{l}\% \text { from } \\
\text { total }\end{array}$ & Median[IQR] & $\begin{array}{l}\text { Mean }[\mathrm{SD}] 95 \% \\
\text { CI }\end{array}$ & $\begin{array}{l}\% \text { from } \\
\text { total }\end{array}$ & \\
\hline $\mathrm{DAP}_{\text {total }}, \mathrm{Gycm}^{2}$ & $\begin{array}{l}236.94 \\
{[186.7]}\end{array}$ & $\begin{array}{l}259.09[ \\
\quad \pm 145.62]\end{array}$ & & $281.20[214.47]$ & $\begin{array}{l}291.23[ \\
\quad \pm 162.59]\end{array}$ & & 0.345 \\
\hline $\mathrm{ED}_{\text {total }}, \mathrm{mSv}$ & $25.82[20.35]$ & $28,24[ \pm 15.87]$ & & $39.84[23.75]$ & $39.59[ \pm 18.5]$ & & $<0.001 *$ \\
\hline $\mathrm{ESD}_{\text {total }}, \mathrm{mGy}$ & $2833[2278]$ & $3036[ \pm 1889]$ & & $2563[3040]$ & $3243[ \pm 2055]$ & & 0.818 \\
\hline $\mathrm{DAP}_{\mathrm{CBCT}}, \mathrm{Gycm} 2$ & n.a & n.a & & $70.76[13,22]$ & $73.85[ \pm 13.53]$ & & n.a \\
\hline $\mathrm{ESD}_{\mathrm{CBCT}}, \mathrm{mGy}$ & n.a & n.a & & $232[43.75]$ & $242.15[ \pm 44.06]$ & & n.a \\
\hline $\mathrm{ED}_{\mathrm{CBCT}}, \mathrm{mSv}$ & n.a & n.a & & $9.19[1.71]$ & $9.6[ \pm 1.75]$ & & n.a \\
\hline $\begin{array}{l}\% \text { of CBCT from total } \\
\text { ED }\end{array}$ & n.a & n.a & & 23.06 & 24.24 & & n.a \\
\hline Number DSA series, $\mathrm{n}$ & $25[15]$ & $28[ \pm 13]$ & & $23[10]$ & $24[ \pm 10]$ & & 0.164 \\
\hline Contrast media, ml & $65[30]$ & $69.2[ \pm 33,2]$ & & $114[40]$ & $122.3[ \pm 25.6]$ & & $<0.001 *$ \\
\hline Fluoroscopy time, min & $23[20]$ & $27.46[ \pm 14.43]$ & & $28[25]$ & $29.74[ \pm 13.44]$ & & 0.265 \\
\hline Procedure time, $\min$ & $70[40]$ & $75.4[ \pm 28]$ & & $120[40]$ & $123[ \pm 33]$ & & $<0.001 *$ \\
\hline $\mathrm{ESD}_{\text {fluoro }}, \mathrm{mGy}$ & $252[282]$ & $337.5[ \pm 289]$ & 11.11 & 634 [603] & $780[ \pm 692]$ & 24.04 & $<0.001^{*}$ \\
\hline $\mathrm{ESD}_{\text {series }}, \mathrm{mGy}$ & 2585 [2047] & $\begin{array}{l}2699.28[ \\
\quad \pm 1735]\end{array}$ & 88.88 & $2245[2675]$ & $\begin{array}{l}2463.59[ \\
\quad \pm 1635]\end{array}$ & 75.96 & 0.5 \\
\hline $\mathrm{DAP}_{\text {fluoro }}, \mathrm{Gycm}^{2}$ & $18.88[24.82]$ & $24.50[ \pm 17.76]$ & 9.46 & $28.98[30.44]$ & $42.57[ \pm 42.14]$ & 14.63 & $0.009^{*}$ \\
\hline $\mathrm{DAP}_{\text {series }}, \mathrm{mGy}$ & $\begin{array}{l}226.75 \\
{[174.5]}\end{array}$ & $\begin{array}{l}234.59[ \\
\quad \pm 145.62]\end{array}$ & 90.54 & 243.46 [206.31] & $\begin{array}{l}248.65[ \\
\quad \pm 132.58]\end{array}$ & 85.37 & 0.593 \\
\hline Bilateral PAE, n (\%) & & $33 / 39$ (84.6) & & & $32 / 39(82.05)$ & & $0.761 * *$ \\
\hline
\end{tabular}

ESD entrance skin dose, DSA digital subtraction angiography, IQR inter quartile ratio, SD standard deviation, DAP dose-area product, CBCT cone-beam computed tomography, $\mathrm{ED}$ effective dose (conversion factor $2 \mathrm{D}-\mathrm{DAP}$ to $\mathrm{ED}: 0.109 \mathrm{mSv} / \mathrm{Gycm}^{2}, \mathrm{DAP} \mathrm{CBCT}_{\mathrm{C}}$ to $\mathrm{ED}: 0.13 \mathrm{mSv} / \mathrm{Gycm}{ }^{2}$ ), ${ }^{*} p$ value are significant different, ${ }^{* *} p$-value calculated with two-sided Fisher's exact test, PAE prostatic artery embolization, \% from total is related to mean values

high rate of technical success in a DSA only protocol. Median total DAP in our study ranged between 236.94 $\mathrm{Gycm}^{2}$ (group A) and $281.20 \mathrm{Gycm}^{2}$ (group B), which is within the range of data published by other authors. Andrade (2017) reported a mean DAP of $450.7 \mathrm{Gycm}^{2}$, and Abt et al. (2018) reported a DAP of $176.5 \mathrm{Gycm}^{2}$ [21, 23].
Mean procedure time in our study ranged from 75,4 $( \pm 28)$ min to $123( \pm 33)$ min, which is comparable to the procedure times of $89.4( \pm 27)$ min reported by Schott et al. [20].

However, magnetic resonance angiography (MRA) can be helpful to determine the origins of PA effectively before 
PAE. Zang et al. reported a sensitivity of $91.5 \%$ and a positive predictive value of $100 \%$ for MRA compared with DSA, and it resulted in a shorter fluoroscopy time and less radiation exposure [24].

The ED is considered the most appropriate quantity for estimating the stochastic risk of exposure to ionizing radiation. However, the complexity of dose calculation for CBCT complicates the comparability of study results. Unfortunately, a variety of conversion factors are available for CBCT because of the heterogeneity of angiographic systems used for acquisition of CBCT, including variations in rotation angles, kilovoltage, fields of views, doses per frame, and rotational speed of acquisition. We used the conversion factor of $0.13 \mathrm{mSv} / \mathrm{Gycm}^{2}$ reported by Suzuki et al., which is the best approximation for the system used in our study [15]. CBCT contributed $23 \%$ of median $\mathrm{ED}_{\text {total }}$ to the procedure in Group B. The median (IQR) ED for CBCT was 9.19 (1.71) $\mathrm{mSv}$, which is lower than values reported by Schott et al. (11.8 $\mathrm{mSv})$, Wang et al. (24 mSv), and Desai et al. (14.6 mSv) [9, 19, 25]. In a recent paper, Zumstein et al. compared the results of 22 studies according to radiation dose for PAE [26]. The overall mean DAP was $181.6 \mathrm{Gycm}^{2}$, resulting in an ED of $28.3 \mathrm{mSv}$. We found an ED of $25.82 \mathrm{mSv}$ for group A and $39.84 \mathrm{mSv}$ for group $\mathrm{B}$, which is within the range reported in the recent literature. Compared with a standard computed tomography scan of the abdomen and pelvis (ED of approximately $11.4 \mathrm{mSv}$ ), the radiation dose for patients undergoing PAE is 2 to 4 times higher [26, 27]. This is comparable to the diagnostic reference level for infrarenal endovascular aortic repair $(32 \mathrm{mSv})$ or trans-arterial chemoembolization of the liver ( $39 \mathrm{mSv}$ ) [27]. The overall risk of radiation-induced cancer death for PAE ranged from $0.107 \%$ to $0.123 \%$ with the highest specific risk for leukemia. Due to significantly lower cell repopulation rate and the reduced life expectancy, these risks become less significant with increasing age [26].

Our study has several limitations. First, we reported only the experience of a single center. Second, no specific conversion factor for our system and procedure is available in the literature, which may have hindered comparability with other studies. Third, the study may have an inherent bias due to the fact that PAE in group A was performed by a single interventionalist with 15 years of experience in embolization therapy, whereas PAE in group B was performed by four interventionalists with experience levels ranging from 4 to 6 years. The learning curve of this procedure affects performance, although we tried to overcome this by excluding data from the first 50 patients in our study, reflecting the results published earlier by our group. [13].

In conclusion, operators with extensive expertise on PAE may not benefit from addition of CBCT to DSA runs, whereas for operators with less expertise, $\mathrm{CBCT}$ when used alongside with DSA runs increased the overall radiation exposure. CBCT accounted for up to $23 \%$ of the total radiation exposure and raised the $\mathrm{CM}$ use of the procedure and should be considered very carefully.

Funding Open Access funding enabled and organized by Projekt DEAL.. This study was not supported by any funding.

\section{Compliance with Ethical Standards}

Conflict of interest The authors declare that they have no conflict of interest.

Ethical Approval All procedures performed in studies involving human participants were in accordance with the ethical standards of the institutional and/or national research committee and with the 1964 Helsinki declaration and its later amendments or compatible ethical standards.

Informed Consent Informed consent was obtained from all individual participants included the study.

Consent for Publication Consent for publication was obtained for every individual person's data included in the study.

Open Access This article is licensed under a Creative Commons Attribution 4.0 International License, which permits use, sharing, adaptation, distribution and reproduction in any medium or format, as long as you give appropriate credit to the original author(s) and the source, provide a link to the Creative Commons licence, and indicate if changes were made. The images or other third party material in this article are included in the article's Creative Commons licence, unless indicated otherwise in a credit line to the material. If material is not included in the article's Creative Commons licence and your intended use is not permitted by statutory regulation or exceeds the permitted use, you will need to obtain permission directly from the copyright holder. To view a copy of this licence, visit http://creativecommons. org/licenses/by/4.0/.

\section{References}

1. Garzon WJ, Andrade G, Dubourcq F, et al. Prostatic artery embolization: radiation exposure to patients and staff. J Radiol Prot. 2016;36:246-54.

2. Pisco JM, Bilhim T, Pinheiro LC, et al. Medium- and long-term outcome of prostate artery embolization for patients with benign prostatic hyperplasia: results in 630 patients. J Vasc Interv Radiol JVIR. 2016;27:1115-22.

3. Kurbatov D, Russo GI, Lepetukhin A, et al. Prostatic artery embolization for prostate volume greater than $80 \mathrm{~cm}^{3}$ : results from a single centre prospective study. Urology. 2014;84:400-4.

4. Franiel T, Aschenbach R, Trupp S, et al. Prostatic artery embolization with $250 \mu \mathrm{m}$ spherical polyzene-coated hydrogel microspheres for lower urinary tract symptoms with follow up MR imaging. J Vasc Interv Radiol. 2018;29:1127-37.

5. Teichgräber U, Aschenbach R, Diamantis I, et al. Prostate artery embolization: Indication, technique and clinical results. RoFo. 2018;190:847-55. 
6. Kaplan SA. Prostate artery embolization is a viable option for treating symptoms of benign prostatic hyperplasia: Con. J Urol. 2017;198:9-11.

7. Carnevale FC, da Motta-Leal-Filho JM, Antunes AA, et al. Qualitiy of life and clinical symptom improvement support prostatic artery embolization for patients with acute urinary retention caused by benign prostatic hyperplasia. J Vasc Int Radiol: JVIR. 2013;24:535-42.

8. Pisco J, Campos Pinheiro L, Bilhim T, et al. Prostatic arterial embolization for benign prostatic hyperplasia: short- and intermediate-term results. Radiology. 2013;266:668-77.

9. Wang MQ, Duan F, Yuan K, et al. Benign prostatic hyperplasia: Cone-beam CT in conjunction with DSA for identifying prostatic arterial anatomy. Radiology. 2017;282:271-80.

10. Bagla S, Rholl KS, Sterling KM, et al. Utility of cone-beam CT imaging in prostatic artery embolization. J Vasc Interv Radiol. 2013;24:1603-7.

11. Chiaradia M, Radelli A, Campeggi A, et al. Automatic threedimensional detection of prostatic arteries using cone-beam CT during prostatic arterial embolization. J Vasc Interv Radiol. 2015;26:413-7.

12. Floridi C, Radaelli A, Abi-Jaoudeh N, et al. C-arm cone-beam computed tomography in interventional oncology: technical aspects and clinical applications. Radiol Med (Torino). 2014;11:521-32.

13. Kriechenbauer BMT, Franiel T, Bürckenmeyer F, et al. Influence of Interventionists expierience on radiation exposure of patients who underwent prostate artery embolization: 4-year results from a retrospective, single-center study. Cardiovasc Intervent Radiol. 2020. https://doi.org/10.1007/s00270-020-02461-1.

14. Struelens L, Vanhavere F, Bacher K, et al. DAP to effective dose conversion in cardiology and vascular/interventional radiology. https://fanc.fgov.be/nl/system/files/report-dap-to-effective-doseconversion-cardiology-vascular-interventional-radiology.pdf.

15. Suzuki S, Furui S, Yamaguchi I, et al. Effective dose during abdominal three-dimensional imaging with a flat-panel detector angiography system. Radiology. 2009;250(2):545-50.

16. Sailer AM, Schurink GWH, Wildberger JE, et al. Radiation exposure of abdominal cone beam computed tomography. Cardiovasc Interv Radiol. 2015;38(1):112-20.

17. Hwang YS, Tsai HY, Lin YY, et al. Investigations of organ and effective dose of abdominal cone-beam computed tomography during transarterial chemoembolization using Monte Carlo simulation. BMC Med Imaging. 2018;18(1):2.

18. De Assis AM, Moreira AM, de Paula Rodrigues VC, et al. Pelvic arterial anatomy relevant to prostatic artery embolization and proposal for angiographic classification. Cardiovasc Intervent Radiol. 2015;38:855-61.

19. Bagla S, Sterling KM. Pitfalls of cone beam computed tomography in prostate artery embolization. Cardiovasc Intervent Radiol. 2014;37(6):1430-5.

20. Schott P, Katoh M, Fischer N, et al. Radiation dose in prostatic artery embolization using cone beam $\mathrm{CT}$ and $3 \mathrm{D}$ roadmap software. J Vasc Interv Radiol. 2019;30(9):1452-8.

21. Andrade G, Khoury HJ, Garzon WJ, et al. Radiation exposure of patients and interventional radiologists during prostate artery embolization: a prospective single-operator study. J Vasc Interv Radiol. 2017;28(4):517-21.

22. Enderlein GF, Lehmann T, von Rundstedt FC, et al. Prostatic artery embolization -Anatomic predictors of technical outcomes. J Vasc Interv Radiol. 2020;31:378-87.

23. Abt D, Hechelhammer L, Müllhaupt G, et al. Comparison of prostatic artery embolization (PAE) versus transurethral resection of the prostate (TURP) for benign prostatic hyperplasia: randomized, open label, noninferiority trial. BMJ. 2018;361:k2338.

24. Zhang JL, Wang MQ, Shen YG, et al. Effectiveness of contrastenhanced MR Angiography for visualization of the prostatic artery prior to prostatic arterial embolization. Radiology. 2019;291:370-8.

25. Desai $\mathrm{H}, \mathrm{Yu} \mathrm{H}$, Ohana E, et al. Comparative analysis of cone beam CT angiogram and conventional angiogram for prostatic artery identification prior to embolization. J Vasc Interv Radiol. 2018;29:229-32.

26. Zumstein V, Binder J, Güsewell S, et al. Radiation exposure during prostatic artery embolization: A systematic review and calculation of associated risks. Eur Urol Focus. 2020. https://doi. org/10.1016/j.euf.2020.04.012.

27. Schegerer A, Loose R, Heuser L, et al. Diagnostic reference levels for diagnostic and interventional X-Ray procedures in Germany: Update and handling. Fortschr Röntgenstr. 2019;191:739-51.

Publisher's Note Springer Nature remains neutral with regard to jurisdictional claims in published maps and institutional affiliations. 